\title{
Fast/Fair Mobile Localization in Infrastructure Wireless
} Sensor Networks

\author{
Kiran Yedavalli ${ }^{\dagger}$, Bhaskar Krishnamachari $^{\dagger}$ \\ $\{k y e d a v a l, b k r i s h n a\} @ u s c . e d u$ \\ Lakshmi Venkatraman ${ }^{\ddagger}$ \\ laksmhi.venkatraman@rtc.bosch.com \\ ${ }^{\dagger}$ Department of Electrical Engineering-Systems, University of Southern California, LA, CA, USA \\ ${ }^{\ddagger}$ Robert Bosch Research and Technology Center, Palo Alto, CA, USA
}

\begin{abstract}
We introduce the problem of fast and fair localization of mobile units in indoor infrastructure wireless sensor networks. We define metrics and derive expressions for delay and fairness of localization and investigate a heuristic algorithm for fast and fair localization. Simulation results show that localization is faster for lower levels of location estimate accuracy, irrespective of anchor density, and that it is fairer for higher anchor densities, irrespective of location estimate accuracy. Also, localization is faster and fairer for grid deployment of anchors as compared to random deployment. The results also suggest that a guarantee on the desired level of location estimate accuracy can be provided for the entire localization area for specific speeds of movement of the mobile unit, and that these speeds are higher for denser anchor deployments.
\end{abstract}

\section{Introduction}

Indoor localization service for mobile devices is an important area of research. In recent years, infrastructure wireless sensor networks (WSN) have emerged as one of the key enablers of this service. For example, the WSN deployed as part of the infrastructure to detect fire can help locate and guide emergency workers during fire emergencies by communicating with the mobile devices carried by them, as illustrated in Figure 1. The wireless devices of the WSN act as anchors, i.e., devices that know their location coordinates, and the mobile devices act as mobile units that need to be localized with the help of anchors. The three main components of any indoor localization service for mobile devices are: (i) accurate localization, (ii) fast localization and (iii) fair localization.

1. Accurate localization: In order to provide finegrained localization in feature-rich areas, such as office buildings and factories, accurate localization is essential. The accuracy of localization depends on the technology used for localization (viz. radio frequency [1], ultra sound [2], etc.) and on the localization algorithm it-self ( $\mathrm{viz}$. range-free ([1],[3],[4]) or range-based ([5],[6])). However, in all localization techniques, the mobile unit needs to communicate with a number of anchors for accurate localization.

2. Fast localization: The response time of the anchors to the mobile unit's localization request places limits on how fast the unit can move while

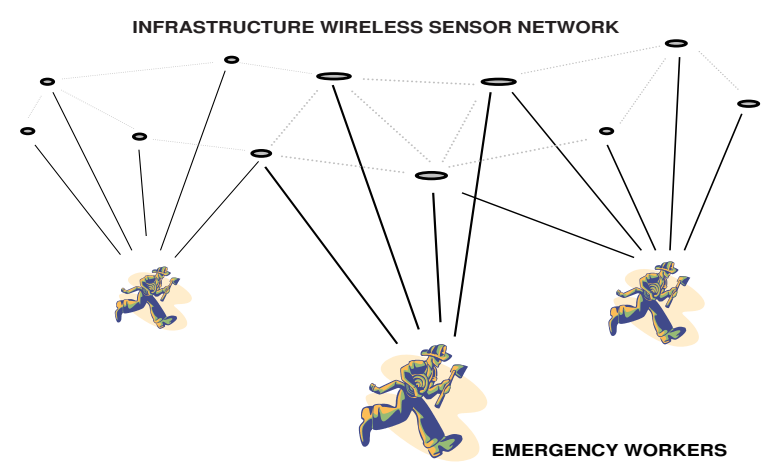

Figure 1: Infrastructure wireless sensor network communicates with mobile devices carried by emergency workers to localize and guide them during an emergency.

obtaining accurate location estimates. The mobile unit should be able to communicate with all the required number of anchors before it moves to its next location. Conversely, the speed of movement of the mobile unit determines the frequency of localization requests and the response time of the anchors should be able to match this frequency. In either case, the response time of the anchors to localization requests should be minimized in order to ensure fast localization.

3. Fair localization: In addition to being fast, the response time of the anchors should not change drastically with the location of the mobile unit in the localization area, i.e., the response time limited speed of movement of the mobile unit should 
not be significantly lower or higher at some locations compared to others. Alternatively, if multiple mobile units at different locations request localization services simultaneously, the response time for all requests should be similar. In order to achieve this, the variation in the anchor response time over all locations of the mobile unit should be minimized.

In this paper, we mainly focus on the problems of fast and fair localization of mobile units. Related work in the literature has studied the speed of the movement of the mobile unit versus the accuracy of tracking [7] and has proposed techniques to balance the speed of tracking and energy consumption [8]. But to the best of our knowledge ours is the first attempt to study the problem of fast and fair localization. We separate the problems of fast and fair localization from the problem of accurate localization so that the solutions for the former are compatible with any solution for the latter. Thus, our work complements the extensive literature of previous works on accurate localization ([1], [2], [3], [4], [5], [6], [9], [10], [11], [12]).

Fast localization requires minimizing the response time of anchors, called the localization delay, to localization requests from mobile units. This requires avoiding collisions and retransmissions of the anchor responses. This can be achieved by splitting time into slots and scheduling anchors to transmit one in each time slot. Fair localization requires minimizing the variation in response time over all locations of the mobile unit in the localization area.

The problems of fast and fair localization can be formulated as a single problem of minimizing the maximum localization delay in the localization area. We show that this problem is closely related to the NP-complete minimum length broadcast frame problem ([13]) in which, the total number of time slots required to schedule all the anchors in the localization area is minimized. We investigate a polynomial time heuristic algorithm for this problem and study its performance in terms of localization delay and fairness. A comparative study is also presented for two different anchor deployment distributions - grid and random - for different anchor density values and different levels of location estimate accuracy.

The rest of the paper is organized as follows: We state our assumptions and introduce terminology, define the different terms used, formulate the problem of fast/fair localization, present the heuristic time slot scheduling algorithm and define metrics to study its performance in Sections II, III, IV, V and VI, respectively. In Section VII, analytical and simulation based evaluations of this algorithm are presented. In Section VIII, realistic application constraints for fast/fair localization are discussed. We conclude and provide directions for future work in Section IX.

\section{Assumptions and Terminology}

In this section we state our assumptions and introduce terminology.

1. The infrastructure wireless sensor network is deployed specifically to provide localization services and it is always powered on ${ }^{1}$. From the perspective of localization services the WSN represents an anchor network and for the rest of the paper it will be referred to as the anchor network.

2. All anchors and mobile units transmit in the same frequency band and at the same power implying that the radio range ( $R$ meters) is the same for all of them.

3. A set $\mathbb{A}$ of $N$ anchors are deployed in a twodimensional, square shaped localization area of side $S(\gg R)$ meters. Their locations depend on the deployment distribution (viz. grid, random, etc). The anchor density, $\beta=\frac{N}{S^{2}}$.

4. The radio range of anchors and mobile units is the same in all directions ${ }^{2}$ and the disc shaped area spanned by the radio range is called a cell. The in-square of a cell is the largest square that is contained within the cell and the out-square of a cell is the smallest square that contains the cell, as illustrated in Figure 2.

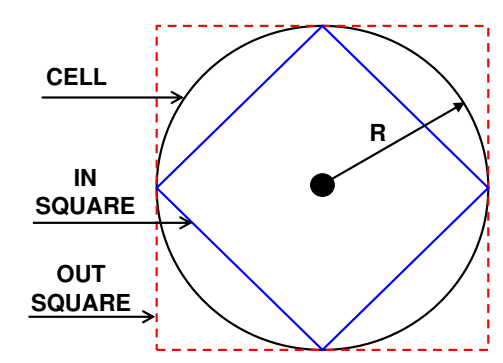

Figure 2: Illustration of a cell, its in-square and outsquare.

\footnotetext{
${ }^{1}$ In section VIII we discuss more realistic cases in which, the WSN has to perform other tasks in addition to localization and is not always powered on.

${ }^{2}$ This is an idealized radio model. In Section VIII we discuss the effects of realistic radio models on fast/fair localization.
} 
5. In order to obtain a finite set of mobile unit locations for evaluation purposes, the locations, $(x, y)$, of the mobile unit are considered to be grid points separated by one meter. In order to avoid edge effects, grid points in a band of $R$ (»1) meters away from the boundaries of the localization area are excluded. Therefore, $(x, y) \in[R, S-R] \times[R, S-R]$.

6. Anchors transmit their location coordinates to the mobile units in localization packets. The mobile units use the localization packets to obtain the anchor node coordinates and to measure the physical quantities required for localization, viz. received signal strength, time of flight, time difference of arrival, angle of arrival, etc. The location estimate accuracy for mobile units increases with number of anchors [1].

7. The number of anchors in the cell of a mobile unit located at $(x, y)$, denoted by $\theta(x, y)$, is determined by the anchor density $(\beta)$, radio range $(R)$ and the mobile unit's location $(x, y)$. On average, the number of anchors in a cell is equal to $\pi R^{2} \beta$. The number of anchors required by a localization technique to guarantee ${ }^{3}$ a desired level of location estimate accuracy, is denoted by $\alpha(\leq \theta(x, y))$.

8. The anchor network is globally synchronized and time is split into slots of equal length. The duration of each time slot $(T)$ consists of the transmission time of a localization packet and its propagation delay, transmission time of any acknowledgment packet and its propagation delay and a guard period to account for time synchronization. For evaluation purposes, $T=20 \mathrm{~ms}$.

9. Let $f$ be any time slot allocation function and let $\mathbf{M}$ (a function of $f$ ) be the number of time slots required by $f$ to allocate time slots to all anchors in the localization area. The $\mathbf{M}$ time slots constitute a time frame and each anchor periodically transmits localization packets based on the position of the time slot allocated to it in the time frame. $f$ is a function that maps the set of anchors $\mathbb{A}$ to the time frame length $\mathbf{M}$, i.e., $f: \mathbb{A} \rightarrow \mathbf{M}$. $\mathbb{F}$ denotes the set of all such allocation functions $f$.

\footnotetext{
${ }^{3}$ It should be noted that in a real scenario, owing to the shadowing and multi-path effects of the wireless channel and features of the localization area, the same number of anchors may not provide the same level of accuracy for all locations of the mobile unit. We assume that the value of $\alpha$ is such that it can guarantee the desired level of accuracy even in the worst case.
}

10. The time slots assigned to anchors in the cell of a mobile unit located at $(x, y)$, are denoted by $t_{i,(x, y)}, i=1, \ldots, \theta(x, y)$.

11. In order to avoid collisions of localization packets, no two anchors within $2 R$ distance of each other are allocated the same time slot. Henceforth, this condition is referred to as the $2 R$-Rule.

\section{Definitions}

1. Localization Request Arrival Time is defined as the time instance when the mobile unit requests the localization service. At this time, the mobile unit starts collecting the localization packets transmitted by the anchors in its cell ${ }^{4}$. Localization requests are assumed to arrive at the starting edges of time slots. Thus, the arrival time, $t$, of a localization request at a mobile unit located at $(x, y)$, is a uniform integer random variable in the interval $[1, \mathbf{M}]$.

2. Localization Delay is defined as the time taken by a certain number of anchors to transmit their localization packets, one each, to the mobile unit. For a given time slot allocation function $f$, it depends on the location of the mobile unit, the localization request arrival time and the number of anchors required for accurate localization. Therefore, it is denoted by $D(x, y, t, \alpha)$. It is measured in time slots.

3. Localizable speed of the mobile unit is defined as the speed at which localization of the desired accuracy is possible. It is determined by the localization delay and time slot duration. The localizable speed $V(x, y, t, \alpha)$ is calculated as follows:

$$
V(x, y, t, \alpha)=\frac{1}{D(x, y, t, \alpha) T} m / s
$$

Since localization delay changes with the location of the mobile unit, the localizable speed of the mobile unit also changes with its location in the localization area.

4. Localization Fairness is defined as the variation in the localizable speed over all possible locations of the mobile unit in the localization area. It is measured as the percentage of locations at

\footnotetext{
${ }^{4}$ Here we assume that anchors transmit their localization packets indefinitely after initialization. In Section VIII we discuss other possible cases.
} 
which the localizable speed is greater than $95 \%$ of its average.

The above terminology is illustrated through an example in Figure 3. It shows $N=36$ anchors deployed in a grid in a square localization area of side $S=5 R$ meters and, their time slots allocated using some time slot allocation function. Notice that the allocation function follows the $2 R-$ Rule and the length of the time frame, $\mathbf{M}$, is equal to 6 for this allocation function. For a mobile unit located at $A$, the number of anchors in its cell $\theta(x, y)=5$ and their respective time slots, $t_{i,(x, y)}$, are $\{1,2,3,4,5\}$. Similarly, the number of anchors in the cell of a mobile unit located at $B$ is 4 and their respective time slots are $\{1,3,4,6\}$. Let the number of anchors required by a particular localization technique for the desired level of accuracy be $\alpha=3$ anchors. If a localization request arrives at time $t=1$ for the mobile unit at $A$ then the localization delay is 3 time slots, where as, if $t=5$, the localization delay is 4 time slots. If the time slot duration is $T=20 \mathrm{~ms}$, then the localizable speeds are $\frac{1}{3 \times 0.02}=16.67 \mathrm{~m} / \mathrm{s}$ and $\frac{1}{4 \times 0.02}=12.5 \mathrm{~m} / \mathrm{s}$ respectively, for the above two localization request arrival times.

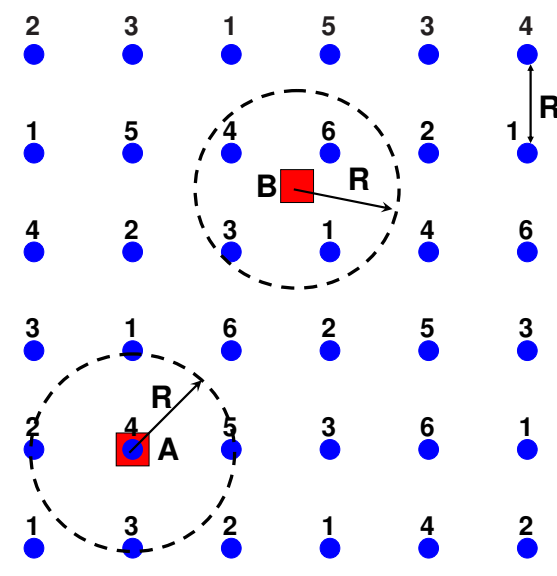

Figure 3: Example illustrating terminology.

\section{Problem Formulation}

The aim of fast/fair localization is minimizing localization delay and maximizing localization fairness. This implies that the time slot scheduling algorithm should minimize localization delay and its standard deviation, over all possible locations of the mobile unit, simultaneously. This can be achieved by minimizing the maximum localization delay over all locations of the mobile unit [14].
In order to normalize the effect of localization request arrival time on localization delay and fairness, the expected value of the localization delay, with respect to $t$, denoted by $E_{t}(D(x, y, t, \alpha))$, is considered.

$$
E_{t}(D(x, y, t, \alpha))=\frac{1}{\mathbf{M}} \sum_{t=1}^{\mathbf{M}} D(x, y, t, \alpha)
$$

Now, the solution to the fast/fair localization problem is an allocation function $f^{*} \in \mathbb{F}$ that minimizes the maximum expected localization delay over all locations $(x, y)$ of the mobile unit, that is:

$$
f^{*}=\arg \min _{f \in \mathbb{F}}\left\{\max _{(x, y)}\left\{E_{t}(D(x, y, t, \alpha))\right\}\right\}
$$

Consider the following two propositions.

\section{Proposition 1.}

$$
\max _{(x, y)}\left\{E_{t}(D(x, y, t, \alpha))\right\} \leq \max _{(x, y, t)}\{D(x, y, t, \alpha)\}
$$

$\forall$ allocation functions $f \in \mathbb{F}$.

Proof. Proof by contradiction. Assume that

$$
\max _{(x, y)}\left\{E_{t}(D(x, y, t, \alpha))\right\}>\max _{(x, y, t)}\{D(x, y, t, \alpha)\}
$$

for some allocation function $f \in \mathbb{F}$. Let $\left(x^{\prime}, y^{\prime}\right) \in$ $[R, S-R] \times[R, S-R]$ be the location at which $E_{t}(D(x, y, t, \alpha))$ is the maximum. Clearly,

$$
\max _{(x, y, t)}\{D(x, y, t, \alpha)\} \geq \max _{t \in[1, \mathbf{M}]}\left\{D\left(x^{\prime}, y^{\prime}, t, \alpha\right)\right\}
$$

From (5) and (6),

$$
E_{t}\left(D\left(x^{\prime}, y^{\prime}, t, \alpha\right)\right)>\max _{t \in[1, \mathbf{M}]}\left\{D\left(x^{\prime}, y^{\prime}, t, \alpha\right)\right\}
$$

This is a contradiction because for all locations $(x, y)$

$$
D(x, y, t, \alpha) \leq \max _{t \in[1, \mathbf{M}]}\{D(x, y, t, \alpha)\}
$$

and from Equation (2), for all locations $(x, y)$

$$
E_{t}(D(x, y, t, \alpha)) \leq \max _{t \in[1, \mathbf{M}]}\{D(x, y, t, \alpha)\}
$$

\section{Proposition 2.}

$$
\max _{(x, y, t)}\{D(x, y, t, \alpha)\} \leq \mathbf{M}
$$

$\forall$ allocation functions $f \in \mathbb{F}$. Recall that $M$ is a function of $f$. 
Proof. From the definition of localization delay, its maximum value is at most equal to the length of the time frame $\mathbf{M}$ for all locations of the mobile unit and for all localization request arrival times.

The above two propositions lead to the following corollary:

\section{Corollary 1.}

$$
\min _{f \in \mathbb{F}}\left\{\max _{(x, y)}\left\{E_{t}(D(x, y, t, \alpha))\right\}\right\} \leq \min _{f \in \mathbb{F}}\{\mathbf{M}\}
$$

Proof. Since inequalities (4) and (10) hold true for all allocation functions $f \in \mathbb{F}$, they are also true for the allocation function $f^{*}$ that minimizes the maximum of the expected localization delay over all locations $(x, y)$ of the mobile unit. Inequality (11) follows from the associative property of inequalities.

Based on the above corollary, the approach we take in this paper for fast/fair localization is to seek an allocation function that minimizes the upper bound on the maximum expected localization delay and study its performance in terms of localization delay and fairness. Now, the solution to the fast/fair localization problem is the allocation function $f^{* *}$ that minimizes the length of the time frame, that is:

$$
f^{* *}=\arg \min _{f \in \mathbb{F}}(\mathbf{M})
$$

The above formulation of the fast/fair localization problem is a flavor of the graph coloring problem called the minimum length broadcast frame problem. Ramaswami et al. in [13] have shown that this problem is NP complete; therefore there is no known polynomial time allocation function $f^{* *}$ that can schedule all anchors in the localization area to transmit in an optimal number of time slots. Nevertheless, many polynomial time heuristic algorithms have been proposed as solutions to this problem. Next, we present and analyze such a heuristic algorithm.

\section{Scheduling Algorithm}

Below, we present pseudo-code for a greedy heuristic algorithm that minimizes the length of the time frame using the location information already programmed into the anchors.

\footnotetext{
A GREEDY HEURISTIC TIME SLOT SCHEDULING ALGORITHM:

Input: Location coordinates $\left\{\left(p_{x i}, p_{y i}\right)\right\}$ of anchors $\left\{q_{i}\right\}$, $i=1, \ldots, N$ in the localization area, reference $(X=$ $(0,0))$ and radio range $(R)$.

Output: Network time slot schedule.
}

$\left\{d_{i}\right\}=\operatorname{DIST}\left(\left\{q_{i}\right\}, X\right)$

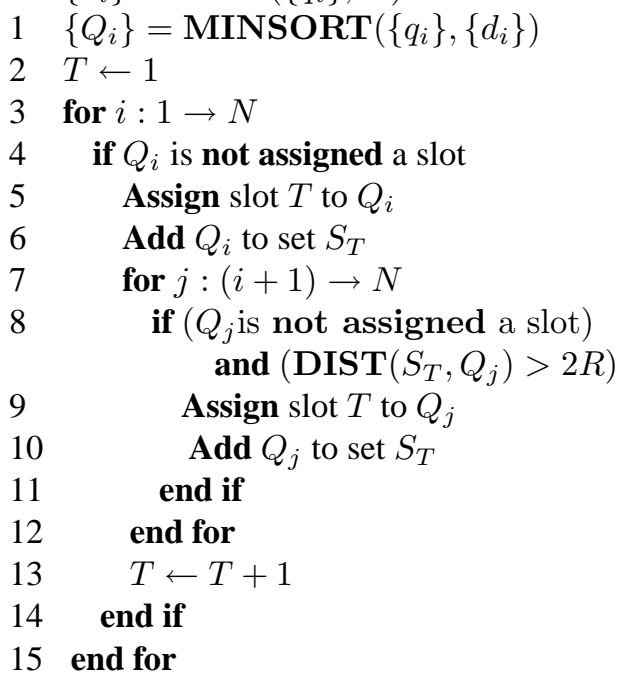

- $\operatorname{DIST}(A, B)$ determines the Euclidean distance between elements of array $A$ and point $B$ and returns the array of distances.

- MINSORT $(A, B)$ minimum sorts the array $A$ based on the values of array $B$ and returns the sorted array.

In a centralized implementation, the above algorithm can be executed on a central server that knows the locations of all anchors in the network; the time slots can be assigned to the anchors later. In a distributed implementation, every anchor executes this algorithm and all of them agree on the same time slot schedule. In this case, every anchor is assumed to know the locations of all other anchors in the network. Complexity Analysis: Every anchor determines the order of all anchors (line 1 of the pseudo code) in the network based on their distances from a reference point (line 0). This takes $O(N \log N)$ time, $O(N)$ space. The ordering of anchors with respect to a reference point in line ensures that anchors that are assigned the same time slot are as close to each other as possible without violating the $2 R$-Rule. This ensures scheduling of anchors in a minimum number of time slots. A time slot is assigned to each anchor in the network in lines $3-15$. This takes $O\left(N^{2}\right)$ time and $O\left(N^{2}\right)$ space. In total, the algorithm takes $O\left(N^{2}\right)$ time and $O\left(N^{2}\right)$ space to assign time slots to all the $N$ anchors in the network.

Next, we define performance metrics for fast/fair localization.

\section{Metrics}

For a given time slot allocation function, localization delay $D(x, y, t, \alpha)$ is determined by the location of 


$$
D(x, y, t, \alpha)= \begin{cases}\tau\left(\alpha, \min \left\{t_{i,(x, y)}\right\}\right)-t+1, & t \leq \min \left\{t_{i,(x, y)}\right\} \\ \tau(\alpha, t)-t+1, & \min \left\{t_{i,(x, y)}\right\}<\max \left\{t_{i,(x, y)}\right\} \cap \alpha \leq \gamma \\ \mathbf{M}+\tau\left(\alpha-\gamma, \min \left\{t_{i,(x, y)}\right\}\right)-t+1, & \min \left\{t_{i,(x, y)}\right\}<t \leq \max \left\{t_{i,(x, y)}\right\} \cap \alpha>\gamma \\ \mathbf{M}+\tau\left(\alpha, \min \left\{t_{i,(x, y)}\right\}\right)-t+1, & t>\max \left\{t_{i,(x, y)}\right\}\end{cases}
$$

Figure 4: Expression for localization delay.

the mobile unit $(x, y)$, the number of anchors in its cell $\theta(x, y)$, their time slots $t_{i,(x, y)}$, the length of the time frame $\mathbf{M}$, the number of anchors required for localization $\alpha$, and the localization request arrival time $t$. This leads to the following proposition.

Proposition 3. Localization delay is given by Equation 13 (see Figure 4). In this equation, $\tau(m, n)$ is the time slot of the $m^{\text {th }}$ anchor starting from time $n$ and $\gamma$ is the number of anchors whose transmission time slots are later than the localization request arrival time $t$.

Proof. All anchors in the cell of the mobile unit located at $(x, y)$ are sorted from the earliest $\left(\min \left\{t_{i,(x, y)}\right\}\right)$ to the latest $\left(\max \left\{t_{i,(x, y)}\right\}\right)$ based on their time slots. Figure 5 shows the time slots of the $\theta(x, y)$ anchors in the cell as a subset of the $\mathbf{M}$ time slots that constitute the time frame. Note that $\left(\min \left\{t_{i,(x, y)}\right\} \geq 1\right)$ and $\left(\max \left\{t_{i,(x, y)}\right\} \leq \mathbf{M}\right)$.

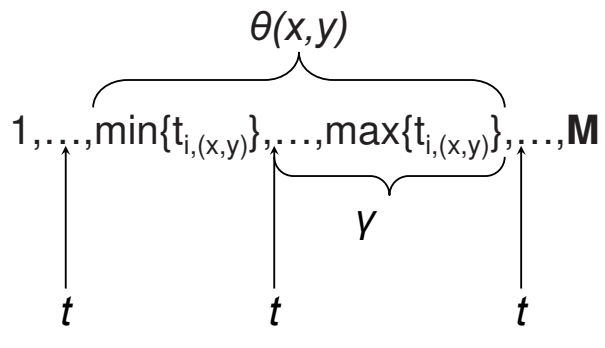

(1)

(2)

(3)

Figure 5: Three different cases (1), (2) and (3) depending on the relative position of localization request arrival time with respect to the times slots of anchors in the cell.

Consider the following three exhaustive cases, based on the position of the localization request arrival time $t$ relative to the set of time slots of anchors in the cell of a mobile unit located at $(x, y)$.

1. The localization request arrival time of the mobile unit is equal to or lower than the minimum of the time slots of the anchors in its cell $(t \leq$ $\left.\min \left\{t_{i,(x, y)}\right\}\right)$ : The mobile unit has to wait till it receives localization packets from all the $\alpha$ anchors. The time slot of the $\alpha^{\text {th }}$ anchor later than $t$ is same as the time slot of the $\alpha^{t h}$ anchor later than $\min \left\{t_{i,(x, y)}\right\}$, which is $\tau\left(\alpha, \min \left\{t_{i,(x, y)\}}\right)\right.$. Thus, the localization delay is given by:

$$
D(x, y, t, \alpha)=\tau\left(\alpha, \min \left\{t_{i,(x, y)}\right\}\right)-t+1
$$

2. The localization request arrival time of the mobile unit is in between the minimum and maximum of the time slots of the anchors in its cell $\left(\min \left\{t_{i,(x, y)}\right\}<t \leq \max \left\{t_{i,(x, y)}\right\}\right)$ : Let $\gamma$ be the number of anchors whose time slots are later than the localization request arrival time $t$. If $\alpha \leq \gamma$, then the mobile unit has to wait till the time slot of the $\alpha^{t h}$ anchor starting from time $t$, which is given by $\tau(\alpha, t)$. Therefore, the localization delay is:

$$
D(x, y, t, \alpha)=\tau(\alpha, t)-t+1
$$

If on the other hand $\alpha>\gamma$, i.e., if the number of anchors with time slots later than the localization request arrival time $t$ is not sufficient, then the mobile unit, after receiving localization packets from the $\gamma$ anchors, has to wait till the end of the frame i.e., time slot $\mathbf{M}$, plus, it has to wait till it receives localization packets from the remaining $\alpha-\gamma$ anchors staring from the first time slot. The time slot of $(\alpha-\gamma)^{t h}$ anchor staring from the first time slot is same as the $(\alpha-\gamma)^{t h}$ anchor staring from time slot $\min \left\{t_{i,(x, y)}\right\}$, which is $\tau\left(\alpha-\gamma, \min \left\{t_{i,(x, y)}\right\}\right)$. Therefore, the localization delay is given by:

$$
D(x, y, t, \alpha)=\mathbf{M}-t+1+\tau\left(\alpha-\gamma, \min \left\{t,{ }_{i,(x, y)}\right\}\right)
$$

3. The localization request arrival time of the mobile unit is later than the maximum of the time slots of the anchors in its cell $(t>$ $\left.\max \left\{t_{i,(x, y)}\right\}\right):$ In this case, the mobile unit has to wait till the end of frame i.e., time slot 
$\mathbf{M}$ and again starting from the first time slot till the time slot of the $\alpha^{\text {th }}$ anchor, which is $\tau(\alpha, \min \{t, i,(x, y)\})$. The localization delay in this case is:

$$
D(x, y, t, \alpha)=\mathbf{M}-t+1+\tau(\alpha, \min \{t, i,(x, y)\})
$$

We consider the following five performance metrics:

1. Average localization delay $\left(D_{\text {avg }}(\alpha)\right)$ : It is the average of the expected localization delay, for each location $(x, y)$ of the mobile unit, over all possible locations of the mobile unit. It is a function of the desired level of accuracy manifested as $\alpha$ and is measured in time slots.

$$
D_{a v g}(\alpha)=\frac{1}{(S-2 R)^{2}} \sum_{x=R}^{S-R} \sum_{y=R}^{S-R} E_{t}(D(x, y, t, \alpha))
$$

2. Average localizable speed $\left(V_{\text {avg }}(\alpha)\right)$ : It is determined by the average localization delay and the length of a time slot and is a function of $\alpha$.

$$
V_{a v g}(\alpha)=\frac{1}{(S-2 R)^{2}} \sum_{x=R}^{S-R} \sum_{y=R}^{S-R} E_{t}(V(x, y, t, \alpha))
$$

where, the expected localizable speed at location $(x, y)$ of the mobile unit, $E_{t}(V(x, y, t, \alpha))$, is given by:

$$
E_{t}(V(x, y, t, \alpha))=\frac{1}{\mathbf{M}} \sum_{t=1}^{\mathbf{M}} V(x, y, t, \alpha)
$$

The units of $V_{\text {avg }}(\alpha)$ are meters/sec. This metric measures the speed of movement of the mobile unit at which it can obtain the desired level of location estimate accuracy, on average, in the localization area. If the mobile unit moves at a speed equal to $V_{\text {avg }}(\alpha)$ there is no guarantee that it will obtain the desired level of accuracy at all locations in the mobile unit.
3. Localization Fairness $(F(\alpha))$ : It measures the percentage of locations of the mobile unit in the localization area that can guarantee the desired location estimate accuracy at a speed of $0.95 V_{\text {avg }}(\alpha)$. Higher the percentage of these locations, higher is the localization fairness.

4. Minimum localizable speed $\left(V_{\min }(\alpha)\right)$ :

$$
V_{\min }(\alpha)=\min _{(x, y)}\left\{E_{t}(V(x, y, t, \alpha))\right\} m / s
$$

This metric measures the localizable speed of the mobile unit at which the anchor network can provide a localization area wide guarantee for the desired level of accuracy. In other words, if the mobile unit moves at a speed that is equal to or lower than $V_{\min }(\alpha)$, it is guaranteed to obtain the desired level of location estimate accuracy for all locations in the localization area.

5. Maximum localizable speed $\left(V_{\max }(\alpha)\right)$ :

$$
V_{\text {max }}(\alpha)=\max _{(x, y)}\left\{E_{t}(V(x, y, t, \alpha))\right\} \mathrm{m} / \mathrm{s}
$$

This metric measures the maximum possible localizable speed of the mobile unit for all locations of the mobile unit in the localization area.

\section{Evaluation}

In this section, first, we analyze the geometries of grid and random deployments of anchors and for each of them derive the upper and lower bounds on the time frame length $\mathbf{M}$ required by any scheduling algorithm (i.e., any allocation function $f$ ). Next, we study the performance of the heuristic algorithm described in Section V in terms of the metrics defined in the previous section using simulations.

\section{VII.A. Analysis}

The definition of a cell ensures that all anchors in the cell of a mobile unit are at most $2 R$ distance away from each other. According to the $2 R-$ Rule described in Section II, the number of time slots required to schedule anchors in the network should be at least equal to the number of anchors in a cell. But this number of time slots is not sufficient. With this understanding, we first analyze the grid deployment of anchors and follow it up with analysis for random deployment. 


\begin{tabular}{|c|c|}
\hline Parameter & Value \\
\hline Radio range, $R$ & 40 meters \\
\hline Localization area side, $S$ & 200 meters \\
\hline Anchor network size, $N$ & $\{121,169,256,324,441\}$ \\
\hline Corresponding anchor densities, & one anchor in \\
$\beta=$ one anchor in $\frac{S^{2}}{N}$ sq. meters & $\{330.6,236.7,156.3,123.5,90.7\}$ sq. meters \\
\hline Number of anchors required for localization, $\alpha$ & $\{3,6,8,10\}$ \\
\hline
\end{tabular}

Table 1: Simulation parameters and their values.

\section{VII.A.1. Grid Deployment}

Proposition 4. For a $2 D$ grid, the number of anchors in the in-square of a cell is $n=2 m^{2}+6 m+5, m=$ $\left(\frac{R}{d}-1\right)$, where $d$ is the inter-node distance and $R$ is the radio range. The number of anchors in the outsquare of a cell is $(2 n-1)$, the number of anchors on the perimeter of the in-square is $(2 \sqrt{2 n-1}-2)$ and the number of anchors on the perimeter of out-square is $(4 \sqrt{2 n-1}-4)$.

We do not provide a formal proof for the above proposition as it can be verified using simple geometric arguments. Notice that, at low anchor densities, the number of anchors in a cell is equal to the number of anchors in its in-square, where as, for high anchor densities, the number of anchors in the cell is greater than the number in its in-square.

Proposition 5. For a $2 D$ grid, $\left(\left\lceil\pi R^{2} \beta\right\rceil+\sqrt{2 n-1}-\right.$ $3)<\mathbf{M}<(2 n-1)$, where $\beta$ is the anchor density.

Proof. If all anchors in the out-square of a cell are assigned different time slots, this schedule satisfies the $2 R$-Rule. Clearly, these number of slots are sufficient. Therefore, according to proposition 4 , at most $(2 n-1)$ time slots are required to schedule all anchors in the localization area.

However, these number of slots are not necessary because there exist pairs of anchors within this square that are greater than $2 R$ distance from each other and these pairs can be assigned the same time slots. In fact, all the slots assigned to anchors on the perimeter of the in-square of the cell can be reused by the anchors on the perimeter of the out-square. And the remaining anchors on the perimeter of the out-square definitely need extra slots. But, the geometry of the cell ensures that these remaining anchors are greater than $2 R$ away from each other in pairs and thus only half of the extra slots are indeed required.

The number of anchors on the perimeter of the cell out-square that do not pair up with the anchors on the perimeter of the cell in-square is $(4 \sqrt{(} 2 n-$ 1) $-4-(2 \sqrt{2 n-1}-2)-4=2 \sqrt{2 n-1}-6)$, where, the extra 4 is subtracted because these many anchors are common between the cell in-square and cell out-square. As stated previously, the number of extra time slots we require is only half the number of anchors that do not pair up, which is $\left(\frac{2 \sqrt{2 n-1}-6}{2}=\right.$ $\sqrt{2 n-1}-3)$ time slots. In total, since the average number of anchors in a cell is $\pi R^{2} \beta$, we need at least $\left(\left\lceil\pi R^{2} \beta\right\rceil+\sqrt{2 n-1}-3\right)$ time slots.

\section{VII.A.2. Random Deployment}

Proposition 6. For uniform random deployment of anchors with density $\beta,\left\lceil\pi R^{2} \beta\right\rceil<\mathbf{M}<\left\lceil 16 R^{2} \beta\right\rceil$.

Proof. As stated previously, the minimum number of time slots required is at least as many as the number of anchors in a cell, which is $\left\lceil\pi R^{2} \beta\right\rceil$.

If all anchors in the out-square of a cell of radius $2 R$ are assigned different time slots and the schedule is repeated through out the network, it is sufficient because, with probability one, no two anchors in the network within $2 R$ distance of each other are assigned the same time slot. The number of time slots required to achieve this is $\left\lceil 16 R^{2} \beta\right\rceil$.

\section{VII.B. Simulations}

The performance of the heuristic scheduling algorithm is measured using simulations in terms of the metrics described in Section VI, for grid and uniform random deployments of anchors for five different anchor density values. Table 1 lists the various simulation parameters and their values.

The anchor locations are generated according to the deployment distribution and the scheduling algorithm uses these locations to assign time slots to them as described in Section V. Table 2 and Table 3 compare the length of the time frame $\mathbf{M}$ with its analytical lower and upper bounds for five different anchor network sizes for grid and uniform random deployments, respectively. For random deployment of anchors the results are averaged over 10 different random anchor network topologies. Clearly, the simulation results are within the analytical bounds. Owing to the regular geometry, the analytical bounds on $\mathbf{M}$ are tighter for grid 


\begin{tabular}{|c|c|c|c|c|}
\hline $\begin{array}{c}\text { Anchor Network } \\
\text { Size, } \boldsymbol{N}\end{array}$ & $\begin{array}{c}\text { Cell In-Square } \\
\text { Size, } n\end{array}$ & $\begin{array}{c}\text { Analytical M } \\
\text { Lower Bound }\end{array}$ & $\begin{array}{c}\text { Simulation } \\
\text { M }\end{array}$ & $\begin{array}{c}\text { Analytical M } \\
\text { Upper Bound }\end{array}$ \\
\hline 121 & $13(m=1)$ & 18 & 19 & 25 \\
169 & $18.5(m=1.5)$ & 25 & 29 & 36 \\
256 & $25(m=2)$ & 37 & 41 & 49 \\
324 & $32.5(m=2.5)$ & 47 & 54 & 64 \\
441 & $41(m=3)$ & 62 & 70 & 81 \\
\hline
\end{tabular}

Table 2: Comparison of analytical lower and upper bounds of $\mathbf{M}$ with simulation results for grid deployment of different value of $N$, the network size. Note that the number of anchors in the in-square of a cell is $n=$ $2 m^{2}+6 m+5, m=\left(\frac{R}{d}-1\right)$ where, $R$ is the radio range and $d$ is the inter anchor distance (Proposition 4 in Section VII.A.1).

\begin{tabular}{|c|c|c|c|}
\hline $\begin{array}{c}\text { Anchor Network } \\
\text { Size, } \boldsymbol{N}\end{array}$ & $\begin{array}{c}\text { Analytical M } \\
\text { Lower Bound }\end{array}$ & $\begin{array}{c}\text { Simulation } \\
\text { Average M }\end{array}$ & $\begin{array}{c}\text { Analytical M } \\
\text { Upper Bound }\end{array}$ \\
\hline 121 & 16 & 29.3 & 75 \\
169 & 22 & 38.3 & 109 \\
256 & 33 & 54.2 & 164 \\
324 & 42 & 66.0 & 208 \\
441 & 56 & 89.6 & 283 \\
\hline
\end{tabular}

Table 3: Comparison of analytical lower and upper bounds of $\mathbf{M}$ with simulation results for random deployment of different value of $N$, the network size. The simulations results are average over 10 different random anchor network topologies.

deployment as compared to random deployment.

Figure 6 plots the simulation results in terms of the five metrics described in Section VI. The main simulation results can be summarized as follows:

1. The average localization delay $\left(D_{\text {avg }}(\alpha)\right)$ increases with the number of anchors required for localization $(\alpha)$, implying that the mobile unit has to wait for a longer time to obtain higher localization accuracy. Also, $D_{\text {avg }}(\alpha)$ is lower for grid deployment as compared to random deployment of anchors and its variation with respect to anchor density is minimal. (Figures 6(a) and $6(\mathrm{~g}))$

2. The average localizable speed $\left(V_{\text {avg }}(\alpha)\right)$ of the mobile unit decreases with the number of anchors required for localization $(\alpha)$, i.e., on average, the mobile unit has to move slower to obtain higher location estimate accuracy, confirming the observation from average localization delay. Also, the average localizable speed of the mobile unit is higher for grid deployment than random deployment of anchors. The effect of anchor density on the average localizable speed is minimal. (Figures 6(c) and 6(f))

3. Localization fairness $F(\alpha)$ is constant with respect to the number of anchors required for localization $(\alpha)$, i.e., localization fairness is independent of the desired level of location estimate accuracy. Also, localization fairness increases with anchor density implying that the number of locations in the localization area that can guarantee the desired level of location estimate accuracy for $95 \%$ of the average localizable speed increases with anchor density. And these locations are higher in number for grid deployment of anchors as compared to random deployment. (Figures 6(b) and 6(h))

4. The minimum localizable speed $\left(V_{\min }(\alpha)\right)$ decreases with number of anchors required for localization $(\alpha)$ and increases with anchor density. This implies that the speed of movement of the mobile unit should be lower for a localization area wide guarantee of higher location estimate accuracy and this speed increases with increasing anchor density. Also, this speed is higher for grid deployment of anchors as compared to random deployment. (Figures 6(d) and 6(f))

5. The absolute maximum localizable speed of the mobile unit over all its locations in the localization area decreases with the desired level of location estimate accuracy. It is higher for random deployment of anchors as compared to grid deployment (even thought the difference between the values for the two distributions is very low) and its dependence on anchor density is minimal. (Figures 6(e) and 6(f)) 


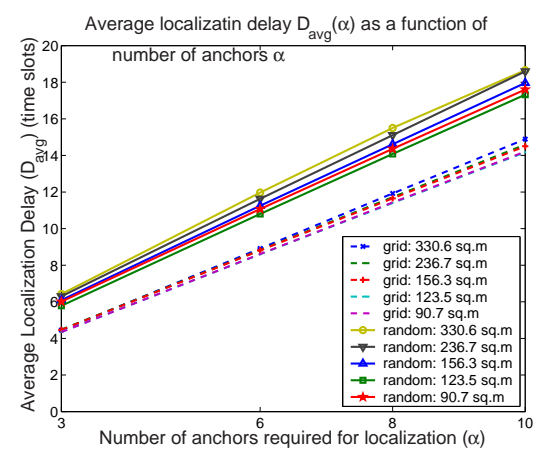

(a)

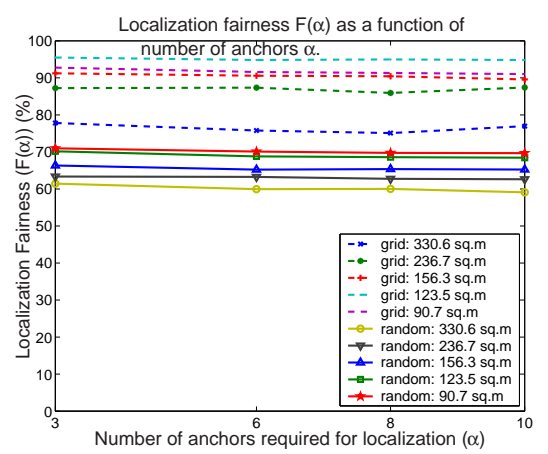

(b)

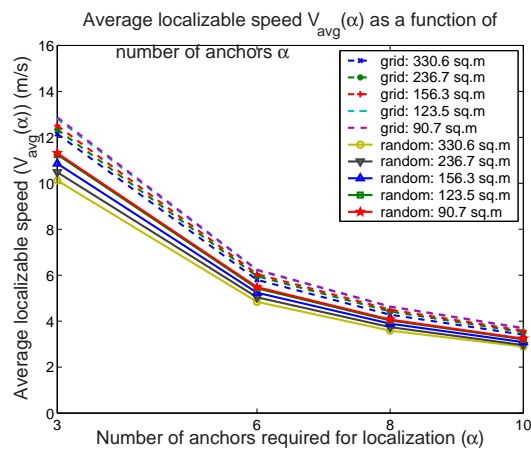

(c)

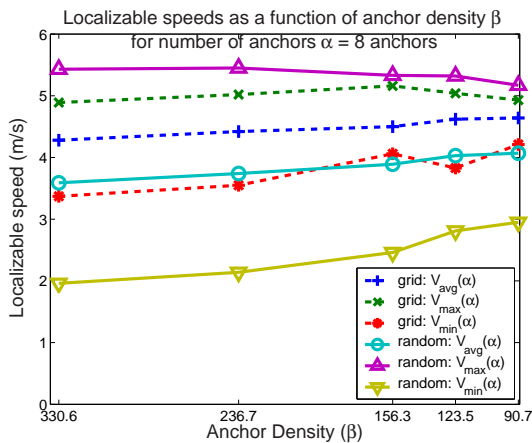

(f)

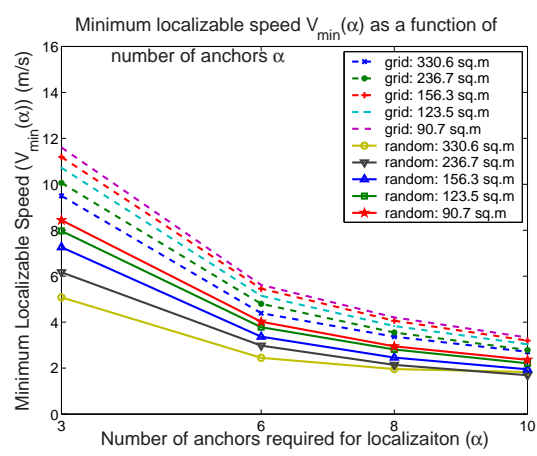

(d)

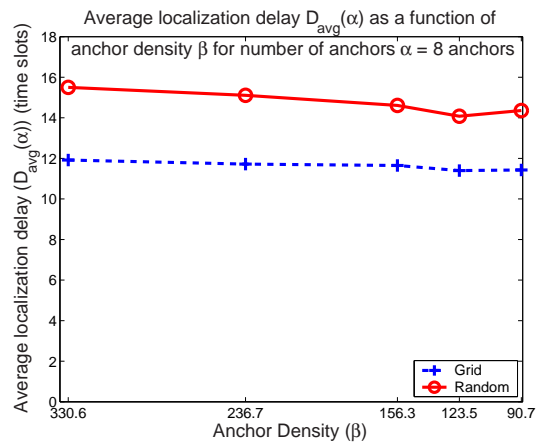

(g)

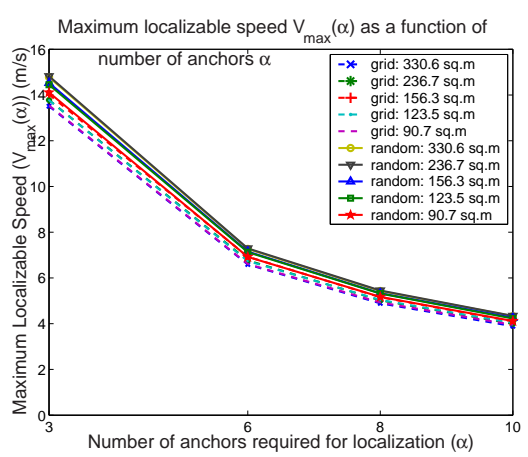

(e)

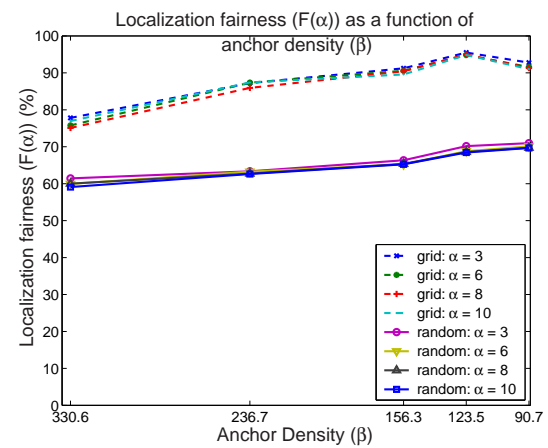

(h)

Figure 6: Simulation Results: (a) Average localization delay $D_{\text {avg }}(\alpha)$, (b) Localization fairness $F(\alpha)$, (c) Average localizable speed $V_{a v g}(\alpha)$, (d) Minimum localizable speed $V_{\min }(\alpha)$, and (e) Maximum localizable speed $V_{\max }(\alpha)$; as a function of number of anchors required for localization $\alpha$, for five anchor density values and for grid and random deployment of anchors. (f) $V_{\text {avg }}(\alpha), V_{\max }(\alpha)$, and $V_{\min }(\alpha)$, (g) Average localization delay $D_{\text {avg }}(\alpha)$; as a function of anchor density $\beta$ for number of anchors required for localization $\alpha=8$, for grid and random deployments of anchors. (h) Localization fairness $F(\alpha)$ as a function of anchor density $\beta$ for four different levels of location estimate accuracy $(\alpha)$ for grid and random deployments of anchors. 


\section{Discussion}

In this section, we present realistic application constraints faced by wireless sensor networks and discuss their effect on fast/fair localization. One of our key assumptions is that the infrastructure wireless sensor network is deployed specifically for localization of mobile units. In reality, this is rarely the case. Infrastructure WSN are expected to provide many services (viz. environment monitoring, event detection, network maintenance etc.) all at the same time. This has a major impact on the performance of localization services as time has to be shared between different services. To determine the exact impact of other WSN services on fast/fair localization the specifications of those services should be known apriori.

Another key assumption in this paper is that the WSN is always powered on; this is not true in reality. The anchor devices are severely energy constrained and therefore they are switched off or are in sleep mode for most of the time. They wake up periodically based on duty cycles that are maintained extremely low, typically $1 \%$, to extend their lifetime. The impact of duty cycles on the metrics of fast/fair localization can be calculated using the actual application dependent duty cycles values.

Since anchors are awake typically only $1 \%$ of the time they need to be woken up for localization when required. When the localization request arrives at the mobile unit, there are two possible network wake up mechanisms:

1. All anchors in the network wake up: Anchors that wake up during the first localization request send out a network wide wake up signal either through a cluster head or through broadcast messages. All anchors in the network wake up as a consequence and start transmitting localization packets periodically according to the time slot schedule determined by the scheduling algorithm. The main advantage of this mechanism is that multiple mobile units can be localized simultaneously and the localization requests that arrive after the network wake up are not subjected to the wake up delay suffered by the first localization request. The main drawback of this mechanism is that, anchors transmit localization packets even when they might not be involved in localization, thus wasting precious energy, unnecessarily.

2. Only anchors in the vicinity of the mobile unit wake up: Only anchors that wake up during the localization request of a mobile unit remain awake and participate in localization. These anchors can transmit their localization packets to the mobile unit according to their time slots assigned by the scheduling algorithm. The main advantage of this scheme is that, anchors spend energy only when it is required. The main disadvantage of this scheme is that all localization requests are subjected to anchor wake up delays.

Clearly, there is a tradeoff in terms of energy and delay between the above two mechanisms that depends on the number of mobile units.

So far, we have assumed an idealistic radio model in which the radio range for anchors and mobile units is the same in all directions. In this idealized radio model, anchors that are farther than $R$ meters from the mobile unit cannot communicate with it. But in reality, localization packets sent by such anchors have a finite probability of reaching the mobile unit [15] and this could potentially lead to collisions of localization packets at the mobile unit. In order to avoid such collisions, the heuristic algorithm could be changed to allocate the same time slot to anchors that are farther than the present distance $(2 R)$.

We have assumed that the anchors and the mobile unit operate in a single frequency band. Instead, if multiple frequencies can be used, the time slot scheduling algorithm can incorporate frequency diversity in addition to time diversity to reduce the response of time of anchors to localization requests and thus reduce the localization delay further. For this, the mobile unit should be able to switch between different frequency bands quickly.

In this paper, we have separated fast/fair localization from the techniques used for accurate localization. In contrast, if the time slot scheduling algorithm takes into account the number of anchors required for a desired level of accuracy by a specific localization technique there could be potential reduction in the localization delay with some collision tolerance at the mobile unit.

\section{Conclusion and Future Work}

In this paper we introduced the problem of fast/fair localization of mobile device in infrastructure wireless sensor networks and showed that it is related to the minimum broadcast frame length problem. We investigated a greedy heuristic time scheduling algorithm for this problem using a defined set of five metrics average localization delay, average localizable speed, localization fairness, minimum localizable speed and maximum localizable speed. We derived lower and 
upper bounds for the number of time slots required to schedule all anchors in the localization area by any scheduling algorithm for grid and random anchor deployment distributions using simple geometric arguments. Next, using simulations, we studied the dynamics of the above five metrics with respect to anchor deployment distributions, anchor densities and location estimate accuracies. Results show that the average localizable speed of mobile unit decreases with increasing level of location estimate accuracy and its dependence on anchor density is minimal. The percentage of locations in the localization area that can guarantee a desired level of location estimate accuracy at a mobile unit speed of $95 \%$ of the average localizable speed, the localization fairness, increases with anchor density and is independent of the accuracy level desired. The average localizable speed of the mobile unit and localization fairness are better for grid deployment of anchors than for random deployment. Also, the localizable speed of the mobile unit at which a localization area wide guarantee of a desired level of accuracy can be provided increases with anchor density and it is higher for grid deployment of anchors.

In the future we plan to study the effect of realistic radio models on the scheduling algorithm and investigate the energy and delay tradeoffs between the two anchor wake-up mechanisms discussed in Section VIII. Also, we wish to study the potential reduction in localization delay, if any, due to incorporation of specific localization algorithms into the time slot scheduling algorithm as discussed in Section VIII. In addition, we would like to explore the performance of contention based schemes in terms of localization delay and fairness, as opposed to scheduling schemes.

\section{Acknowledgements}

We wish to thank Abtin Keshavarzian and Bhaskar Srinivasan of Robert Bosch Research and Technology Center, Palo Alto, CA, for many valuable discussions.

\section{References}

[1] Kiran Yedavalli, Bhaskar Krishnamachari, Sharmila Ravula, and Bhaskar Srinivasan. Ecolocation: A Sequence Based Technique for RF Localization in Wireless Sensor Networks. In The Fourth International Conference on Information Processing in Sensor Networks (IPSN 2005), Los Angeles, CA, April 2005.

[2] Nissanka B. Priyantha, Anit Chakraborty, and Hari Balakrishnan. The Cricket Location-Support System. In ACM MOBICOM, Boston, MA, August 2000.
[3] N. Bulusu, J. Heidemann, and D. Estrin. Gps-less low-cost outdoor localization for very small devices. IEEE Personal Communications Magazine, October 2000.

[4] T. He, B.M. Blum C. Huang, J.A. Stankovic, and T. Abdelzaher. Range-Free Localization Schemes for Large Scale Sensor Networks. In Mobicom, San Diego, CA, September 2003.

[5] N. Patwari and A.O. Hero III. Using Proximity and Quantized RSS for Sensor Localization in Wireless Networks. In WSNA, San Diego, CA, September 2003.

[6] Kiran Yedavalli. Location Determination Using IEEE 802.1lb. Master's thesis, The University of Colorado at Boulder, December 2002.

[7] Adam Smith, Hari Balakrishnan, Michel Garaczko, and Nissanka Bodhi Priyantha. Tracking Moving Devices with the Cricket Location System. In $2 n d$ International Conference on Mobile Systems, Applications and Services (Mobisys 2004), Boston, MA, June 2004.

[8] Sameer Tilak, Vinay Kolar, Nael B. Abu-Ghazaleh, and Kyoung-Don Kang. Dynamic Localization Protocols for Mobile Sensor Networks. In IEEE International Workshop on Strategies for Energy Efficiency in Ad-hoc and Sensor Networks (IEEE IWSEEASN'05), Phoenix, AZ, USA, April 2005.

[9] A. Nasipuri and K. Li. A Directionality based Location Discovery Scheme for Wireless Sensor Networks. In WSNA, Atlanta, Georgia, September 2002.

[10] C. Savarese, J.M. Rabaey, and J. Beutel. Locationing in Distributed Ad-Hoc Wireless Sensor Networks. In ICASSP, May 2001.

[11] Andreas Savvides, H. Park, and M.B. Srivastava. The Bits and Flops of the N-hop Multilateration Primitive For Node Localization Problems. In WSNA, Atlanta, Georgia, September 2002.

[12] A. Savvides, C.C. Han, and M.B. Srivastava. Dynamic Fine Grained Localization in Ad-Hoc Sensor Networks. In Proceedings of the Fifth International Conference on Mobile Computing and Networking, Mobicom 2001, pages 166-179, Rome, Italy, July 2001.

[13] Rajiv Ramaswamy and Keshab K. Parhi. Distributed Scheduling of Broadcasts in a Radio Network. In INFOCOM '89, volume 2, pages 407-504, Ottawa, Ont., Canada, April 1989.

[14] Dimitri Bertsekas and Robert Gallager. Data Networks (Second Edition). Prentice Hall, 1991.

[15] Marco Zuniga and Bhaskar Krishnamachari. Analyzing the Transitional Region in Low Power Wireless Links. In First IEEE International Conference on Sensor and Ad hoc Communications and Networks (SECON), Santa Clara, CA, October 2004. 